\title{
Patterns of contraceptive use among young Australian women with chronic disease: findings from a prospective cohort study
}

\author{
Melissa Harris ( $\square$ melissa.harris@newcastle.edu.au ) \\ University of Newcastle https://orcid.org/0000-0002-5733-9684 \\ Nicholas Egan \\ UoN: University of Newcastle \\ Peta M Forder \\ UoN: University of Newcastle \\ Deborah Bateson \\ Family Planning New South Wales \\ Aaron L Sverdlov \\ UoN: University of Newcastle \\ Vanessa E Murphy \\ UoN: University of Newcastle \\ Deborah Loxton \\ UoN: University of Newcastle
}

\section{Research Article}

Keywords: chronic disease, contraceptive methods, long-acting reversible contraception, pill, withdrawal, young women, cohort study, longitudinal

Posted Date: October 29th, 2021

DOI: https://doi.org/10.21203/rs.3.rs-1007064/v1

License: (c) (i) This work is licensed under a Creative Commons Attribution 4.0 International License. Read Full License 


\section{Abstract \\ Background}

Given chronic disease is increasing among young women and unintended pregnancies among these women are associated with poor maternal and fetal outcomes, these women would benefit from effective preconception care. However, there is a lack of understanding of how these women use or don't use contraception to inform such interventions. This study examined patterns of contraceptive use among an Australian cohort of young women, with and without chronic disease, and investigated factors which influenced contraceptive use over time.

\section{Methods}

Using data from 15,244 young women from the Australian Longitudinal Study on Women's Health (born 1989-95), latent transition analysis was performed to identify distinct contraceptive patterns among women who were at risk of an unintended pregnancy. Multinomial mixed-effect models were used to evaluate the relationship between contraceptive combinations and chronic disease.

\section{Results}

Differences in contraceptive use over time were found for women with cardiac disease, those with autoinflammatory diseases. Compared to women without chronic disease using the pill alone, women with cardiac disease had double the odds of using 'other' low efficacy contraception and condoms ( $\mathrm{OR}=2.2,95 \% \mathrm{Cl}$ : 1.34-3.59) and a modest increase in the odds of using the combined oral contraceptive pill and condoms $(\mathrm{OR}=1.39,95 \% \mathrm{Cl}$ : 1.03-1.89). Women with autoinflammatory disease had increased odds of using LARC and condoms (OR=1.58, 95\% Cl: 1.04-2.41), using 'other' low efficacy contraception and condoms (OR=1.69, 95\% Cl: 1.11-2.57), and using combined oral contraceptive pill and condom use (OR=1.38, 95\% Cl: 1.09-1.75), compared to women without chronic disease using the pill. There was little evidence to suggest that contraceptive patterns differed for women with asthma or diabetes from women without chronic disease.

\section{Conclusion}

The findings identified a need for effective contraceptive counselling as part of routine chronic disease care and improved communication between healthcare providers and women with chronic disease to improve young women's contraceptive knowledge and agency in contraceptive choice, particularly for those with cardiac or autoinflammatory conditions. This may be the key to reducing high-risk unintended pregnancies among this vulnerable population.

\section{Plain English Summary}

Chronic disease is increasing among young women and unintended pregnancies among these women are associated with poor outcomes for both the mother and baby. To optimise outcomes, it is important for these women to plan pregnancies and use effective contraception until such time. However, there is a lack of understanding of how these women use or don't use contraception, particularly with respect to highly effective contraception. This study examined patterns of contraceptive use among an Australian cohort of young women (born 1989-95), with and without chronic disease, and investigated factors which influenced contraceptive use over time. We found differences in contraceptive use over time for women with cardiac disease and those with autoinflammatory diseases. Importantly, compared to women without chronic disease using the pill alone, women with cardiac disease had double the odds of using low efficacy contraception. While women with autoinflammatory disease were $69 \%$ more likely to using long-acting methods combined with condoms, these women were also $70 \%$ more likely use low efficacy contraception, compared to women without chronic disease who used the pill only. Contraceptive patterns did not differ for women with asthma or diabetes from women without chronic disease. The findings identified a need for effective contraceptive counselling as part of routine chronic disease care and improved communication between healthcare providers and women with 
chronic disease to improve young women's contraceptive knowledge and contraceptive decision-making, particularly for those with cardiac or autoinflammatory conditions. This may be the key to reducing high-risk unintended pregnancies among this vulnerable population.

\section{Background}

Access to, and use of, effective contraception is the cornerstone of preconception care for women, allowing autonomous control over fertility and reproductive decisions. Although Australia has high access to contraception, unintended pregnancy remains an important public health issue, with around $40 \%$ of pregnancies unintended at conception [1]. All women of reproductive age may experience an unintended pregnancy, however certain sub-populations have been found to be at increased risk. There is mounting evidence to suggest that women with chronic disease experience unintended pregnancy at a higher rate than women without chronic disease, with rates in this population reported as high as $60 \%$ [2, 3]. For women with chronic disease, unintended pregnancies are associated with serious adverse maternal and perinatal outcomes, including congenital abnormalities, pre-term labour, spontaneous abortion, premature birth and fetal death [4]. Optimised preconception care and reproductive life planning is therefore critical to the prevention of unintended pregnancies and reduction in pregnancy-related complications for these women.

Despite this, there is a lack of high-quality evidence regarding how young women with chronic disease use, or don't use, contraception, particularly in Australia. Of the few available international studies, the findings have been equivocal, in part due to their cross-sectional, retrospective nature and concentration on single disease entities with small samples [3,5-7]. Crosssectional studies fail to capture the dynamic nature of contraceptive use in different contexts over time, with contraceptive patterns found to vary according to a range of sociodemographic, lifestyle and sexual and reproductive health factors [8-11]. Additionally, none have specifically focused on women in early adulthood, the time of highest unintended pregnancy risk. In one of the only longitudinal studies available, only one-third of women with chronic disease (hypertension, asthma, hypothyroidism, diabetes, obesity, rheumatoid arthritis [RA], inflammatory bowel disease [IBD], or systemic lupus erythematosus [SLE]) [12] were found to be users of prescription contraception during the three-year observation period compared to $41 \%$ of women without chronic disease. This study, however, was limited by a short time frame and reliance on insurance claims data. More recently, cross-sectional analysis of population-level data in the U.S. found substantially higher rates of contraceptive use among women of reproductive age with diabetes, cardiovascular disease, or asthma (87\%) and when focused on contraceptive efficacy, contraceptive use differed by chronic disease type. Importantly, women with diabetes and cardiovascular disease were more likely to be users of less effective methods than women without chronic disease [13].

Chronic disease is on the rise among women of reproductive age in Australia (and increasing with successive generations) [14]. Further, contraceptive patterns differ markedly by age, with younger women reporting higher use of multiple (often less effective) methods than older women $[15,16]$. This underscores the need to examine contraceptive patterns that better reflect 'actual' contraceptive practices. It is therefore imperative to use nationally representative population-level data to understand how contraceptive use changes over time among women with chronic disease to prevent unintended pregnancy in this high-risk population. This study aimed to examine patterns of contraceptive use among an Australian cohort of women born 1989-95 with and without chronic disease and determine factors that influence these contraceptive behaviours over time.

\section{Methods}

\section{Study design}

Data were obtained from the 1989-95 cohort of the Australian Longitudinal Study on Women's Health (ALSWH), a national population-based study examining health and wellbeing among Australian women. Specific recruitment methods have been described in detailed elsewhere [17]. Briefly, women from this cohort were recruited through an open recruitment strategy involving a mix of online and offline methods including paid Facebook advertising, promotion using social and other internetbased media, paid and unpaid promotion through traditional media, and peer referral. Women were eligible for inclusion if they were aged 18-23 years in 2012-2013, had a Medicare number (Australia's universal health insurance scheme) and consented to their survey data being linked to administrative health data. Women recruited through these methods were found to be

Page $3 / 20$ 
demographically representative of similarly aged women in the Australian population, except for an overrepresentation of Australian-born and tertiary-educated women [18]. After the baseline survey in 2012-13, online surveys were deployed annually to 2017, with another survey deployed in 2019.

\section{Participants}

This analysis focused on women from the 1989-95 cohort who completed Surveys 1, 3 or 5 conducted in 2012-13 (aged 18-24 years), 2015 (aged 20-26 years) and 2017 (aged 22-28 years). Of the 17,010 women who completed the baseline survey, 15,376 women were eligible for linked data analysis and completed the questions related to contraceptive use (Figure 1). Women were considered not at risk of an unintended pregnancy at each time point if they reported being currently pregnant, trying to become pregnant, or if their partner could not have children. Based on these criteria, 132 women were not at risk of a future unintended pregnancy at all three time points, resulting in a final sample of 15,244 women ( $90 \%$ of the original cohort).

\section{Measures}

\section{Contraceptive use}

Participants were asked to report their contraceptive use the last time they had vaginal sex from a list of six options: "the pill"; "condoms"; "Implanon" (i.e., progestogen-only implant); “Mirena” (i.e., progestogen IUD); "other contraceptive"; and "none”. At Survey 1 , women were also provided the opportunity to expand on their response to "other contraceptive" as part of a free-text option. For the purposes of analysis, the progestogen-only implant and progestogen IUD were combined into a single "hormonal LARC" item.

\section{Chronic disease}

The presence or absence of seven physical chronic diseases that have been found to be relatively common among women of reproductive age and have been associated with poor maternal and perinatal outcomes were examined [4]. These included diabetes, cardiac disease (including hypertension), asthma, autoinflammatory arthropathies and connective tissue disease, IBD, multiple sclerosis, and thyroid disease. Disease ascertainment was assessed using multiple data sources and employing disease-specific algorithms (developed in concert with clinical experts) to increase chronic disease accuracy. Detailed information on the methods employed are described elsewhere [14]. Briefly, case ascertainment was achieved using survey and linked administrative health data, including individual state and territory-based Admitted Patient Data Collections (APDC) for hospital admissions; the Pharmaceutical Benefits Scheme (PBS) for prescribed medications, and the Medicare Benefits Schedule (MBS) for disease-specific medical claims. "Cases" were required to have either (a) one or more indication(s) in either the APDC or MBS; (b) indication in two or more ALSWH surveys; or (c) two or more disease-related prescriptions within a 12-month period, reported in two separate calendar years. Where a medication had multiple indications for treatment, medication ascertainment was used if there was an indication for that condition from another data source (e.g., was also captured through MBS or survey data).

\section{Covariates}

Sociodemographic variables included age (years), highest educational qualification (year 12 or below; certificate/diploma; university), area of residence (categorised according to the ARIA+ classification system as major cities; inner regional; outer regional/remote/very remote), relationship status (partnered; unpartnered), employment status (full-time; part-time; not in paid work), country of birth (Australia; other English speaking; other), and ability to manage on available income (impossible/difficult all the time; difficult some of the time; not too bad/easy). Possession of a Health Care Card (a concession card for governmentsubsidised healthcare) was also included as a surrogate for socioeconomic status (yes; no).

Health-related factors included smoking status (current smoker; non-smoker), alcohol consumption (non-drinker; low risk drinker; infrequent drinker; risky/high risk drinker) [19] and body mass index (BMl: underweight $\left[<18.5 \mathrm{~kg} / \mathrm{m}^{2}\right]$; healthy weight $[\geq 18.5$ and $<25 \mathrm{~kg} / \mathrm{m}^{2}$ ]; overweight [ $\geq 25$ and $<30 \mathrm{~kg} / \mathrm{m}^{2}$ ]; obese [ $\left.\geq 30 \mathrm{~kg} / \mathrm{m}^{2}\right]$ ) [20]. Psychological distress was measured using the Kessler 10 (K10) scale with scores aggregated into categories (low [scores 10-15]; moderate [scores 16-21]; high [scores 22-29]; very high [scores 30-50]) [21]. 
Reproductive health factors included pregnancy history (yes; no), history of pregnancy termination (yes; no) and history of miscarriage (yes; no). Given contraceptive methods are often used for non-contraceptive reasons, we also adjusted for the presence or absence of self-reported gynaecological conditions such as polycystic ovarian syndrome (yes; no), endometriosis (yes; no), and the experience of menstrual symptoms such as irregular periods, heavy period, or severe period pain 'often' (yes; no).

\section{Statistical analysis}

Latent transition analysis was used to group women into latent statuses over time based on reported contraceptive combinations [15]. Contraceptive data were entered into separate latent transition models with three to eight latent statuses each to determine the combinations of contraceptive use that best fit the data. A classify-analyse approach was used to assign each participant to a latent status at each time point, according to the latent status with the greatest posterior probability. Latent transition analysis was performed using PROC LTA procedure (The Methodology Centre, Penn State) in SAS 9.4 software. Multinomial mixed-effect models using generalised structural equation modelling was then developed in Stata 15.1, with the assigned latent status describing a particular contraceptive behaviour as the multinomial outcome, and time-varying predictors (including an indicator for chronic disease). Correlation between observations was accounted for by treating participant ID as a random intercept. Separate models were conducted for the presence of any chronic disease and each of the chronic diseases.

\section{Results}

\section{Sample characteristics}

At baseline in $2013,18.9 \%$ of women reported having at least one of the physical chronic diseases of interest, and by 2017 , this had increased to $22.6 \%$ (Table 1). The most prevalent chronic disease reported at baseline was asthma (14.3\%), with the prevalence of diabetes, cardiac disease, autoinflammatory arthropathies and connective tissue disease, IBD, multiple sclerosis and thyroid disease all less than $5 \%$. By 2017, the prevalence of asthma had increased to $16.1 \%$, and slight increases were observed for all other conditions. Given the low frequencies of autoinflammatory conditions (i.e., autoinflammatory arthropathies and connective tissue disease, IBD, multiple sclerosis, and thyroid disease), these were combined into a single autoinflammatory disease category for subsequent modelling.

Table 1

Proportion of women with chronic disease over the observation period.

\begin{tabular}{|c|c|c|c|c|c|c|}
\hline \multirow[t]{2}{*}{ Chronic disease } & \multicolumn{2}{|c|}{ Survey 1 (2013) } & \multicolumn{2}{|c|}{ Survey 3 (2015) } & \multicolumn{2}{|c|}{ Survey 5 (2017) } \\
\hline & $\mathbf{n}$ & $(\%)$ & $\mathbf{n}$ & $(\%)$ & $\mathbf{n}$ & $(\%)$ \\
\hline Any chronic disease & 2,674 & $(18.9)$ & 1,707 & $(21.8)$ & 1,628 & $(22.6)$ \\
\hline Diabetes & 388 & $(2.7)$ & 289 & $(3.7)$ & 282 & $(3.9)$ \\
\hline Cardiac disease & 277 & $(2.0)$ & 206 & $(2.6)$ & 208 & $(2.9)$ \\
\hline Asthma & 2,028 & $(14.3)$ & 1,238 & $(15.8)$ & 1,160 & $(16.1)$ \\
\hline Autoinflammatory arthropathies & 179 & $(1.3)$ & 122 & $(1.6)$ & 116 & $(1.6)$ \\
\hline Inflammatory bowel disease & 56 & $(0.4)$ & 48 & $(0.6)$ & 52 & $(0.7)$ \\
\hline Thyroid disease & 145 & $(1.0)$ & 112 & $(1.4)$ & 122 & $(1.7)$ \\
\hline Multiple sclerosis & 13 & $(0.1)$ & 18 & $(0.2)$ & 22 & $(0.3)$ \\
\hline Autoinflammatory disease ${ }^{b}$ & 405 & $(2.9)$ & 302 & $(3.9)$ & 297 & $(4.1)$ \\
\hline $\begin{array}{l}\text { Note: } 2013(\mathrm{n}=14,150) ; 2015(\mathrm{n}= \\
\text { a Includes autoinflammatory arth }\end{array}$ & $17(n=7$ & $y b c$ & Ca & id di & ad $n$ & scle \\
\hline
\end{tabular}


At baseline in 2013 (aged 18-24 years), there were few differences in characteristics between women with and without physical chronic disease, except for income management, BMI, and the experience of menstrual symptoms (Table 2). Women with chronic disease were more likely to report their ability to manage on available income as impossible or difficult always (30.7\% vs. $25.4 \%$ ). Women with chronic disease were also less likely to report being in the healthy BMI category than women without chronic disease (52.5\% vs $60.0 \%$ ) and were more likely to report menstrual symptoms often ( $42.6 \%$ vs $35.9 \%$ ). 
Table 2

Baseline characteristics of women aged 18-24 years (in 2013), according to chronic disease status $(n=14,150)$

\begin{tabular}{|c|c|c|c|}
\hline \multirow[t]{4}{*}{ Characteristic } & \multirow[t]{4}{*}{ Category } & \multicolumn{2}{|c|}{ Presence of chronic disease } \\
\hline & & No & Yes \\
\hline & & $n=11,476$ & $n=2,674$ \\
\hline & & $\mathrm{n}(\%)$ & $\mathrm{n}(\%)$ \\
\hline \multicolumn{4}{|l|}{ Sociodemographics } \\
\hline \multirow[t]{4}{*}{ Country of birth } & Australia & $10,472(91.3)$ & $2,512(93.9)$ \\
\hline & Other English-speaking background & $414(3.6)$ & $71(2.7)$ \\
\hline & Non-English-speaking background & $434(3.8)$ & $65(2.4)$ \\
\hline & Missing & $156(1.4)$ & $26(1.0)$ \\
\hline \multirow[t]{4}{*}{ Area of residence } & Major cities & $8,616(75.1)$ & $1,960(73.3)$ \\
\hline & Inner regional & $1,935(16.9)$ & $514(19.2)$ \\
\hline & Outer regional/remote/very remote & $921(8.0)$ & $199(7.4)$ \\
\hline & Missing & $4(0.0)$ & $1(0.0)$ \\
\hline \multirow[t]{4}{*}{ Education } & Year 12 or below & $5,615(48.9)$ & $1,286(48.1)$ \\
\hline & Certificate/diploma & $3,238(28.2)$ & $817(30.6)$ \\
\hline & University & $2,616(22.8)$ & $571(21.4)$ \\
\hline & Missing & $7(0.1)$ & $0(0.0)$ \\
\hline \multirow[t]{3}{*}{ Relationship status } & Partnered & $3,572(31.1)$ & $914(34.2)$ \\
\hline & Non-partnered & $7,898(68.8)$ & $1,759(65.8)$ \\
\hline & Missing & $6(0.1)$ & $1(0.0)$ \\
\hline \multirow[t]{4}{*}{ Work status } & None & $2,291(20.0)$ & $642(24.0)$ \\
\hline & Part-time & $6,233(54.3)$ & $1,401(52.4)$ \\
\hline & Full-time & $2,939(25.6)$ & $630(23.6)$ \\
\hline & Missing & $13(0.1)$ & $1(0.0)$ \\
\hline \multirow[t]{4}{*}{ Income management } & Impossible/difficult always & $2,912(25.4)$ & $821(30.7)$ \\
\hline & Difficult sometimes & $4,162(36.3)$ & $1,000(37.4)$ \\
\hline & Not too bad/easy & 4,393 (38.3) & $851(31.8)$ \\
\hline & Missing & $9(0.1)$ & $2(0.1)$ \\
\hline \multirow[t]{3}{*}{ Health care card status } & No & $7,838(68.3)$ & $1,638(61.3)$ \\
\hline & Yes & $3,631(31.6)$ & $1,035(38.7)$ \\
\hline & Missing & $7(0.1)$ & $1(0.0)$ \\
\hline \multicolumn{4}{|l|}{ Health factors } \\
\hline Smoking status & Non-smoker & $9,063(79.0)$ & $2,084(77.9)$ \\
\hline
\end{tabular}




\begin{tabular}{|c|c|c|c|}
\hline \multirow[t]{4}{*}{ Characteristic } & \multirow[t]{4}{*}{ Category } & \multicolumn{2}{|c|}{ Presence of chronic disease } \\
\hline & & No & Yes \\
\hline & & $n=11,476$ & $n=2,674$ \\
\hline & & n (\%) & $\mathrm{n}(\%)$ \\
\hline & Current smoker & $2,413(21.0)$ & $590(22.1)$ \\
\hline & Missing & $0(0)$ & $0(0)$ \\
\hline \multirow[t]{5}{*}{ Alcohol consumption } & Non-drinker & $563(4.9)$ & $175(6.5)$ \\
\hline & Low risk drinker & $6,813(59.4)$ & $1,427(53.4)$ \\
\hline & Infrequent drinker & $3,621(31.6)$ & $964(36.1)$ \\
\hline & Risky/high risk drinker & $479(4.2)$ & $108(4.0)$ \\
\hline & Missing & $0(0)$ & $0(0)$ \\
\hline \multirow[t]{5}{*}{ Body mass index } & Underweight & $904(7.9)$ & $152(5.7)$ \\
\hline & Healthy weight & $6,882(60.0)$ & $1,403(52.5)$ \\
\hline & Overweight & $2,080(18.1)$ & $570(21.3)$ \\
\hline & Obese & $1,307(11.4)$ & $486(18.2)$ \\
\hline & Missing & $303(2.6)$ & $63(2.4)$ \\
\hline \multirow[t]{5}{*}{ Psychological distress (K10) } & Low & $2,474(21.6)$ & $445(16.6)$ \\
\hline & Moderate & $3,380(29.5)$ & $716(26.8)$ \\
\hline & High & $3,152(27.5)$ & $787(29.4)$ \\
\hline & Very high & $2,469(21.5)$ & $726(27.2)$ \\
\hline & Missing & $1(0.0)$ & $0(0.0)$ \\
\hline \multicolumn{4}{|l|}{ Reproductive health } \\
\hline \multirow[t]{3}{*}{ History of pregnancy } & No & $9,883(86.1)$ & $2,211(82.7)$ \\
\hline & Yes & 1,589 (13.8) & $463(17.3)$ \\
\hline & Missing & $4(0.1)$ & $O(0)$ \\
\hline \multirow[t]{3}{*}{ History of termination } & No & $10,696(93.2)$ & $2,481(92.8)$ \\
\hline & Yes & $765(6.7)$ & $191(7.1)$ \\
\hline & Missing & $15(0.1)$ & $2(0.1)$ \\
\hline \multirow[t]{3}{*}{ History of miscarriage } & No & $10,938(95.3)$ & $2,494(93.3)$ \\
\hline & Yes & $521(4.5)$ & $177(6.6)$ \\
\hline & Missing & $17(0.1)$ & $3(0.1)$ \\
\hline \multirow[t]{3}{*}{ Parity } & Zero & $11,072(96.5)$ & $2,517(94.1)$ \\
\hline & One & $312(2.7)$ & $115(4.3)$ \\
\hline & Two & $76(0.7)$ & $37(1.4)$ \\
\hline
\end{tabular}




\begin{tabular}{|c|c|c|c|}
\hline \multirow[t]{4}{*}{ Characteristic } & \multirow[t]{4}{*}{ Category } & \multicolumn{2}{|c|}{ Presence of chronic disease } \\
\hline & & No & Yes \\
\hline & & $n=11,476$ & $n=2,674$ \\
\hline & & n (\%) & $\mathrm{n}(\%)$ \\
\hline & Three or more & $16(0.1)$ & $5(0.2)$ \\
\hline & Missing & $0(0.0)$ & $0(0.0)$ \\
\hline \multirow[t]{3}{*}{ Menstrual symptoms } & No & $7,353(64.1)$ & $1,536(57.4)$ \\
\hline & Yes & $4,123(35.9)$ & $1,138(42.6)$ \\
\hline & Missing & $0(0)$ & $0(0)$ \\
\hline \multirow[t]{3}{*}{ History of PCOS* } & No & $11,116(96.9)$ & $2,489(93.1)$ \\
\hline & Yes & $360(3.1)$ & $185(6.9)$ \\
\hline & Missing & $0(0)$ & $0(0)$ \\
\hline \multirow[t]{3}{*}{ History of endometriosis } & No & $11,224(97.8)$ & $2,573(96.2)$ \\
\hline & Yes & $252(2.2)$ & $101(3.8)$ \\
\hline & Missing & $0(0)$ & $0(0)$ \\
\hline
\end{tabular}

\section{Trends in contraceptive use}

In 2013, the proportion of women using some form of contraception at the time of their last vaginal sex was similar for women with (85.5\%) and without physical chronic disease (86.7\%) (Table 3), with similar proportions observed in 2017. By 2017, there was lower use of the oral contraceptive pill and condoms, although use of hormonal LARC had increased, with a noticeable increase in the use of the progestogen IUD. In 2013, use of the progestogen IUD was relatively low for women with and without physical chronic disease (3.0\% vs. $1.8 \%$ respectively), but had a similar rise in both groups of women by $2017(10.8 \%$ vs. $8.8 \%$ respectively). 
Table 3

Contraceptive trend over time by chronic disease status.

\begin{tabular}{|c|c|c|c|c|c|}
\hline \multirow[t]{4}{*}{ Contraceptive } & & \multicolumn{2}{|c|}{$\begin{array}{l}\text { Chronic disease status } \\
2013 \text { (Survey } 1 \text { ) }\end{array}$} & \multicolumn{2}{|c|}{$\begin{array}{l}\text { Chronic disease status } \\
2017 \text { (Survey 5) }\end{array}$} \\
\hline & & No & Yes & No & Yes \\
\hline & & $n=11,476$ & $n=2,674$ & $n=5,589$ & $n=1,628$ \\
\hline & & $\mathrm{n}(\%)$ & $\mathrm{n}(\%)$ & $\mathrm{n}(\%)$ & $\mathrm{n}(\%)$ \\
\hline Any contraception & & $10108(88.1)$ & $2339(87.5)$ & $4953(88.6)$ & $1430(87.8)$ \\
\hline Pill & & $6343(55.3)$ & $1465(54.8)$ & $2522(45.1)$ & $693(42.6)$ \\
\hline Condom & & $4950(43.1)$ & $1135(42.4)$ & $2090(37.4)$ & $592(36.4)$ \\
\hline Progestogen-only implant & & $1188(10.4)$ & $283(10.6)$ & $671(12.0)$ & $202(12.4)$ \\
\hline Progestogen IUD & & $212(1.8)$ & $81(3.0)$ & $493(8.8)$ & $176(10.8)$ \\
\hline Other methods & & $68(0.6)$ & $10(0.4)$ & $247(4.4)$ & $106(6.5)$ \\
\hline No contraception & & $1368(11.9)$ & 335 (12.5) & $636(11.4)$ & $198(12.2)$ \\
\hline \multirow[t]{4}{*}{ Number of contraceptives } & 0 & $1368(11.9)$ & $335(12.5)$ & $636(11.4)$ & $198(12.2)$ \\
\hline & 1 & $7244(63.1)$ & $1632(61.0)$ & $3897(69.7)$ & $1093(67.1)$ \\
\hline & 2 & $2832(24.7)$ & $701(26.2)$ & $1045(18.7)$ & $336(20.6)$ \\
\hline & $3+$ & $32(0.3)$ & $6(0.2)$ & $11(0.2)$ & $1(0.1)$ \\
\hline
\end{tabular}

Among women with chronic disease, $61.0 \%$ and $67.1 \%$ reported using only one contraceptive method in 2013 and 2017 respectively. The proportion of women who reported two or more contraceptive methods declined from one-quarter in 2013 to one-fifth in 2017. Around 12\% of women reported not using any contraception in both 2013 and 2017, irrespective of chronic disease status.

\section{Contraceptive combinations}

The optimal LTA model was selected based on clinical interpretability, latent class separation and $\mathrm{G}^{2}$, AIC and BIC (Supplementary Table S1). We also sought to minimise the number of time points with very low membership probabilities ( $<2 \%)$ as this would contribute to numerical estimation issues in subsequent regression models. A six-status model was determined to be the optimal model for categorising complex contraceptive use, based on goodness-of-fit statistics and clear clinical interpretability. The six-status model was preferred over the five-status model, which exhibited two very similar latent statuses that both featured condoms and the pill. The seven-status model was unviable due to low status membership probabilities.

As presented in Table 4, the first status (described as "Condom") was characterised by high condom use (100\% probability) but included some supplementation with the oral contraceptive pill (6\% probability). The second status (described as "Pill and condom") was dominated by both the pill ( $91 \%$ probability) and condom use ( $94 \%$ probability). The third status (described as "Pill") was dominated by use of the oral contraceptive pill (100\% probability) with a low probability of condom use (10\% probability). The fourth status, termed "LARC and condom", included women predicted to use a hormonal LARC (100\% probability) but also supplemented with condoms (19\% probability). The fifth status was termed "Other and condom", which included women predicted to use other contraceptive methods ( $100 \%$ probability) and/or condoms (18\% probability), while the sixth status (described as "None") captured the absence of any active contraceptive methods. Across all three time points in the six-status model, Status 3 ("Pill") was the most prevalent contraceptive status and for this reason was selected as the reference class for subsequent analysis. 
Table 4

Six-status model for contraceptive patterns over time for the 1989-95 ALSWH cohort.

\begin{tabular}{|c|c|c|c|c|c|c|}
\hline \multirow[t]{2}{*}{ Latent Status } & \multirow[t]{2}{*}{ Latent status description } & \multicolumn{5}{|c|}{ Item-response probabilities for each status } \\
\hline & & Condom & Pill & aLARC & Other & None \\
\hline Status 1 & Condom & 1.00 & 0.06 & - & - & - \\
\hline Status 2 & Pill and condom & 0.91 & 0.94 & - & - & \\
\hline Status 3 & Pill & 0.10 & 1.00 & - & - & - \\
\hline Status 4 & LARC and condom & 0.19 & 0.03 & 1.00 & - & - \\
\hline Status 5 & Other and condom & 0.18 & 0.04 & - & 1.00 & \\
\hline Status 6 & None & - & - & - & - & 0.95 \\
\hline \multicolumn{7}{|c|}{$\begin{array}{l}\text { Note: Dashed cells have probability }<0.01 \text {. Shaded status (\#3, Pill) was selected as the reference class for subsequent } \\
\text { analyses. }\end{array}$} \\
\hline \multicolumn{7}{|c|}{$\begin{array}{l}\text { a LARC refers to the use of hormonal long-acting reversible contraception (progestogen-only implant and the progestogen } \\
\text { IUD). }\end{array}$} \\
\hline \multicolumn{7}{|c|}{ Copper IUD is included as part of "other" contraception. } \\
\hline
\end{tabular}

\section{Contraceptive use by women with chronic disease}

Following the adjustment for confounders, the presence of any physical chronic disease was associated with increased odds of using other contraception and condoms ( $\mathrm{OR}=1.29,95 \% \mathrm{Cl}=1.07$ to 1.57$)$, compared to use of the pill alone (Table 5). When focused on the relationship between specific chronic diseases and contraceptive use over time, women with cardiac disease had increased odds of combined pill and condom use ( $\mathrm{OR}=1.39,95 \% \mathrm{Cl}=1.03$ to 1.89$)$, as well as no contraception (OR=1.54, $95 \%$ $\mathrm{Cl}=1.10$ to 2.16 ), compared to the use of the pill alone. Notably, there was more than a 2-fold increase in the odds of using other contraception and condoms observed for women with cardiac disease ( $\mathrm{OR}=2.20,95 \% \mathrm{Cl}=1.34$ to 3.59). Women with autoinflammatory disease had increased odds of LARC and condoms $(\mathrm{OR}=1.58)$, increased odds of other contraception and condoms $(\mathrm{OR}=1.69)$ and increased odds of combined pill and condom use $(\mathrm{OR}=1.38)$ when compared to use of the pill alone. There was little evidence to suggest that contraceptive use was influenced by a diagnosis of diabetes or asthma. 
Table 5

Multinomial mixed-effect models for the effect of chronic disease status on contraceptive use for Australian women, aged 18 to

28 across three time points $(2013,2015 \& 2017)$.

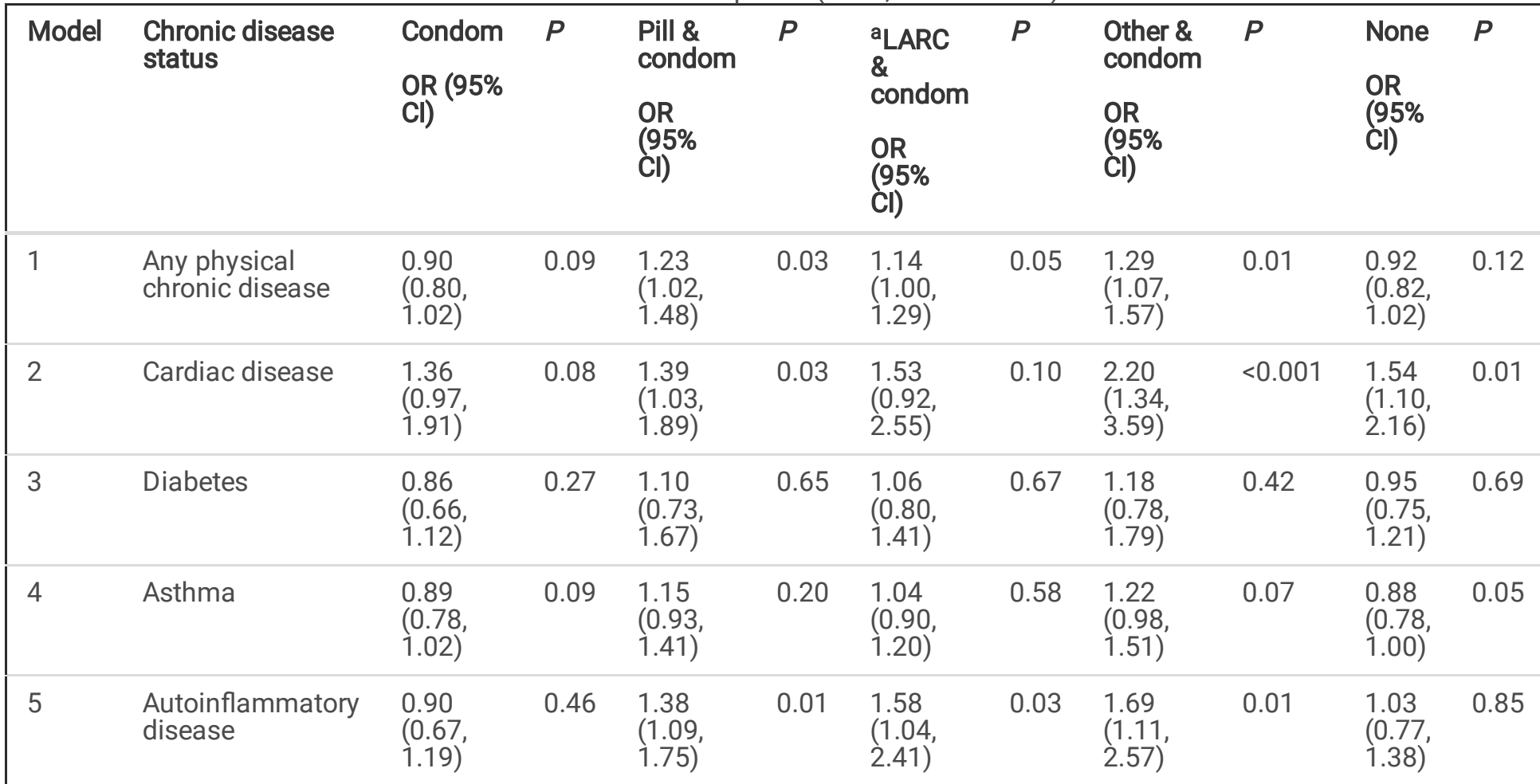

Reference latent status = Status 4 (“Pill”); reference level for chronic disease status = disease not present.

a LARC refers to the use of hormonal long-acting reversible contraception (progestogen-only implant and the progestogen IUD). Copper IUD is included as part of "other" contraception.

Each model controlled for age, country of birth, area of residence, highest educational qualification, work status, managing on available income, smoking status, alcohol consumption, body mass index, psychological distress, history of pregnancy, history of termination, history of miscarriages, menstrual symptoms, history of polycystic ovary syndrome, history of endometriosis, and survey wave.

\section{Discussion}

Young women with chronic disease were more likely to engage in the use of low efficacy contraception over the observation period compared to women in the general population, despite demonstrating similar rates of contraceptive use. When specific chronic diseases were examined, this pattern of use was evident for women with cardiac and autoinflammatory conditions but not those with diabetes or asthma. This study therefore provides much needed insight into how young Australian women with chronic disease 'actually' use contraception at the population level. The findings have important implications for the delivery of contraceptive counselling and reproductive life planning for young women with chronic disease, especially those with cardiac and autoinflammatory diseases.

Overall, contraceptive use among women meeting our chronic disease definitions was found to be high across the five-year observation period (above 85\%). Although we focused exclusively on young women and included the ability to examine both hormonal and non-hormonal contraceptive methods, we found self-reported hormonal-based contraceptive use among women with chronic disease to be substantially higher than that reported by DeNoble et al. [12]. Further, while our prevalence of contraceptive use among women with chronic disease was similar to that reported in a 2016 cross-sectional U.S. study, they found that the use of effective and highly effective contraception was lowest among women aged 18-24 years ( $<45 \%)$ with highly effective contraceptive use driven largely by sterilisation (even for young women) [13]. In contrast, high efficacy contraceptive use in our study was attributed to the use of hormonal LARC. This finding is important given that LARC use has previously been reported as low among young women in Australia [10,15,22]. Our finding may be reflective of increased awareness in Australia around the acceptability of LARC across the reproductive life course and in particular the suitability of 
LARC for young nulliparous women. Increased LARC use is now also recognised as a key indicator in meeting the priorities of the current Australian National Women's Health Strategy 2020-2030 [23].

Although our study findings demonstrate that LARC use is increasing among young women, when we examined patterns of contraceptive use, only women diagnosed with autoinflammatory disease were found to have $58 \%$ increased odds of hormonal LARC use compared to women without chronic disease using the pill. While this finding is promising given that LARC are recommended as first line options for women with autoinflammatory disease (including those on immunosuppressants) and provide the greatest protection against unintended pregnancy $[9,24]$, they contrast with a number of studies which have demonstrated low LARC uptake among this chronic disease population $[25,26]$. Concerningly, while women with autoinflammatory conditions were more likely to use LARC than other women, they also had a $69 \%$ increase in odds of using low efficacy methods compared to women without chronic disease using the pill. Our finding is supported by research showing that women with SLE discontinue hormonal contraception (mainly the combined oral contraceptive pill) following diagnosis and take up lower efficacy methods despite being on potentially teratogenic medications (including methotrexate) [27]. Use of low efficacy methods with high typical use failure rates, place these women at significant risk of unintended pregnancy. Importantly, withdrawal and condoms have been found to be the most prevalent forms of contraception used by women with SLE and RA including a substantial number with unintended pregnancy histories [28]. Most concerning, however, is that young women diagnosed with cardiac disease had $220 \%$ and $54 \%$ increased odds of using low efficacy methods and of being non-users of contraception, compared to women without chronic disease using the pill, respectively. This finding is supported by previous cross-sectional research $[6,13]$. Our findings, coupled with the emerging body of literature around contraceptive use among cardiac and autoinflammatory disease point to an unmet need regarding evidence-based contraceptive advice and support, particularly from rheumatologists and cardiologists who are responsible for prescribing (potentially teratogenic) medication and monitoring disease activity.

In our study no discernible difference in the contraceptive patterns between women with diabetes compared to women without chronic disease using the pill were found. While the international evidence around this topic has been equivocal, Australian research has found that although women with diabetes are high users of contraception (mostly condoms and the oral contraceptive pill), contraception is not consistently used [29]. This is important given that the pill and condoms are the most prevalent forms of contraception used at the time of unintended pregnancy among young Australian women [30]. Therefore, although the combined oral contraceptive pill is not contraindicated for young women with uncomplicated diabetes, increasing the use of highly effective contraception among this population is still warranted given the need for engagement in preconception contraceptive care to prevent adverse maternal and perinatal consequences associated with unintended pregnancy [31]. International research has shown only $32 \%$ of teens and $18 \%$ of young adults with type 1 diabetes attain recommended glycaemic control, yet only one-quarter of adolescents are aware of the adverse impacts associated with poor glycaemic control in pregnancy [32].

While young women with chronic disease should be supported to choose and use a method of contraception that aligns with their reproductive and personal goals, our findings point to an underutilisation of highly effective LARC among most young women with chronic disease and suggest that gaps in the delivery of preconception contraceptive counselling may exist in Australia, particularly for those with cardiac and autoinflammatory conditions. Low rates of general and disease-specific contraceptive counselling among chronic disease populations have been demonstrated internationally, even in the presence of potentially teratogenic medication [33-35]. The reason for low contraceptive counselling among women with chronic disease however is not well understood. It has been postulated that lack of both contraceptive use and contraceptive counselling among women with chronic disease is attributed to misperceptions around fertility, knowledge regarding pregnancy risks [36, 37] and health system factors whereby the chronic condition takes up the healthcare providers' time and focus during appointments [38]. As a result, women with chronic disease often receive minimal contraceptive counselling from either general practitioners (GPs) or specialists. It has also been argued that healthcare providers are uncomfortable with prescribing contraception to women they perceive to be at higher risk of adverse events due to lack of familiarity with the safety of different methods for women with different medical conditions [39]. 
The contraceptive needs of women with cardiac disease are especially challenging to navigate due to variability in potential risks associated with both contraception type and the nature and severity of the cardiac disease. In general, however, medical eligibility guidelines for the provision of contraception [24], indicate the risks associated with the use of estrogen-containing hormonal contraception (e.g., combined oral contraceptive pill) outweigh the benefits given the increased risks of arterial and venous thrombosis for a number of cardiac conditions, including severe or poorly controlled hypertension (although in practice, the combined oral contraceptive pill is generally only advised against when there is a history of unprovoked arterial/venous thrombosis or a known genetic defect). By contrast, progestogen-only methods are not associated with a risk of venous or arterial thromboembolic disease and are safer options for most women with cardiac disease (including those with congenital heart disease) $[40,41]$. Of the progestogen-only methods available, the progestogen-only implant followed by levonorgestrel-IUDs have the highest efficacy against pregnancy. Levonorgestrel IUDs are a suitable choice for women with cardiac conditions (including those with congenital heart disease), and their effect on reducing menstrual bleeding can be beneficial, including for those on anticoagulant therapy. However, women with major cardiac disease may require cardiology input before insertion [24]. Similarly, in the presence of autoinflammatory conditions such as RA and IBD, there are concerns associated with estrogencontaining contraception in relation to disease exacerbation as well as thrombotic effects (particularly with women who have antiphospholipid syndrome and a history of IBD-related surgery or past biologic therapy use). Potential malabsorption issues for women with IBD will also limit the use of combined hormonal and progestogen-only oral methods.

Given the exposure to medications used in the treatment of autoinflammatory conditions, chronic hypertension and other cardiac diseases are associated with major congenital malformations [42-44], young women with chronic disease (particularly those with cardiac and autoinflammatory conditions) therefore require individualised contraceptive counselling and reproductive life planning based on their specific condition(s), with consideration around disease severity and medication use [24]. As these women are already engaged within the healthcare system this provides an ideal opportunity to provide such care as part of a well-coordinated structured approach to chronic disease management involving GPs and specialists where contraceptive conversations are routine. While some key bodies such as the Australian Rheumatology Association provide guidance on prescribing medications during pregnancy and recommend that women of child-bearing age receive preconception counselling and discussions around contraception, Australia currently lacks formal guidelines for autoinflammatory diseases as well as other chronic conditions. Increased access to, and awareness of current therapeutic guidelines by peak medical associations and key bodies (e.g., Therapeutic Guidelines) as well as development of referral pathways are required alongside increasing medical education given there is a demonstrated lack of expertise and confidence regarding the provision of family planning among GPs and specialists in Australia and internationally [45-47]. This will ensure that young women with chronic disease receive access to clear and accurate information and regarding their contraceptive options. Increased education for young women with chronic disease about the risks of unintended pregnancy and the benefits of appropriate highly effective contraception for their specific condition, as well as evidence-based information to dispels myths around LARC including its impact on future fertility are also required [48]. Importantly for young women who develop chronic disease early in life, greater acknowledgement, information provision and screening of paediatric populations in relation to sexual activity and contraceptive needs is needed [49]. Such practices also need to be maintained as young women transition from paediatric to adult services to ensure these women do not fall through the gap.

A key strength of this study was the ability to examine contraceptive patterns for women diagnosed with seven key chronic diseases during early adulthood using longitudinal data. In addition, we were able to apply statistical techniques to accurately identify contraceptive combinations. This improves on previous research which has examined contraception within the context of chronic disease as either users or non-users or has used hierarchical approaches [12,13]. Our analysis shows that for young women contraceptive use is complex and requires examination of all contraceptive combinations. A further strength of the study is the methods used to ascertain chronic disease cases with the inclusion of both survey and administrative data providing the ability to capture all forms of chronic disease [14]. No studies have previously utilised these approaches for examining chronic disease among women of reproductive age. Given that contraceptive use and risk of an unintended pregnancy is dynamic across the reproductive life course [16], we excluded women not at risk of a future unintended pregnancy at each of the time points. Few studies have accounted for this in longitudinal research [50]. 
While we were able to examine contraceptive use at yearly intervals, the questions regarding contraceptive use were not substantive after survey 1 . Unfortunately, we were not able to accurately ascertain the use of methods such as the depot injection or copper IUD. Due to minimal self-reports of their use, these items were included as 'other' contraception. As such, we have potentially over-estimated the use of low efficacy methods. Additionally, while we examined contraception over time using latent transition analysis, to examine time-varying covariates (including chronic disease status) we employed a classify-analyse approach. We acknowledge that this approach may induce some measurement error due to the uncertainty in latent status classification [51].

\section{Conclusion}

This study demonstrated that young Australian women with chronic disease take up contraception at similar rates to their same aged peers in the community, although they are more likely to use less effective contraception, particularly women with cardiac and autoinflammatory diseases. Our findings highlight the need for all young women with chronic disease to have the opportunity for comprehensive contraceptive counselling as part of their routine chronic disease management, at diagnosis and at regular ongoing appointments, to ensure they are aware of the risks of unintended pregnancy and are provided with the highest efficacy contraceptive options that are most appropriate to their specific circumstances. To achieve this, increasing contraceptive knowledge and awareness of the need for contraceptive counselling among specialists and improvement in communication between GPs and specialists as part of a well-coordinated teams-based approach to chronic disease management is required. This will not only increase women's agency in contraceptive knowledge and choices but also reduce high-risk unintended pregnancies in this vulnerable population.

\section{List Of Abbrevations}

\begin{tabular}{|ll|}
\hline Rheumatoid arthritis & RA \\
\hline Inflammatory bowel disease & IBD \\
\hline Systemic lupus erythematosus & SLE \\
\hline Australian Longitudinal Study on Women's Health & ALSWH \\
\hline Admitted Patient Data Collection & APDC \\
\hline Medical Benefit Schedule & MBS \\
\hline Pharmaceutical Benefits Scheme & PBS \\
\hline Body Mass Index & BMI \\
\hline Long-acting reversible contraception & LARC \\
\hline General practitioner & GP \\
\hline Intrauterine device & IUD \\
\hline
\end{tabular}

\section{Declarations}

\section{Ethics approval and consent to participate}

All data for this project were obtained from the ALSWH (see www.alswh.org.au/ for further details), approved under their Expression of Interest process (EOI A696) and provided in de-identified form. This project has ongoing ethical clearance from the University of Newcastle and University of Queensland's Human Research Ethics Committees. Ethical approval for linkage of ALSWH survey data to the Admitted Patients Data Collections (APDC) was received from the NSW Population and Health Services Research Ethics Committee and other equivalent state and territory-based committees. Linkages to the National Death Index (NDI) and Australian Cancer Database (ACD) were approved by the Australian Institute of Health and Welfare Ethics Committee. Women provided explicit written consent to participate in the ALSWH as well as for linkage to de-identified 
administrative health records prior to 2005 . From 2005, an 'opt-out' consent process was approved by the data custodians and relevant ethics committees for data linkage, with participants regularly reminded of this process. ALSWH participants who decline health record linkage are excluded from data linkage requests.

\section{Consent for publication}

Not applicable.

\section{Availability of data and materials}

The data used as part of this analysis are bound by ethical restrictions due to containing person level data. These restrictions have been imposed by the ALSWH data access committee. A core survey dataset is available through the Australian Data Archive (please visit https://dataverse.ada.edu.au/dataverse/alswh_core_release for further information about obtaining these data). For further information regarding full access to Australian Longitudinal Study on Women's Health data, including linked datasets, requests are to be sent to info@alswh.org.au.

\section{Competing interests}

The authors declare that they have no competing interests

\section{Funding}

Dr Melissa Harris is funded through an Australian Research Council Discovery Early Career Researcher Award (DE190101134). Dr Aaron Sverdlov is supported by a Future Leader Fellowship (Award ID 101918) from the National Heart Foundation of Australia. Dr Vanessa Murphy is funded through a Medical Research Future Fund Investigator Grant (APP1196252).

\section{Author contributions}

MLH was responsible for the study concept. MLH, NE and PMF contributed to the study design and analysis plan. NE conducted the analysis. MLH, PMF, NE, ALS, VEM contributed to case ascertainment data acquisition. MLH drafted the manuscript. All author revised the manuscript for intellectual content. All authors approved the final version of the paper and take responsibility for its content

\section{Acknowledgements}

The research on which this paper is based was conducted as part of the Australian Longitudinal Study on Women's Health by the University of Newcastle and the University of Queensland. We are grateful to the Australian Government Department of Health for funding and to the women who provided the survey data.The authors acknowledge the Department of Health and Medicare Australia for providing MBS and PBS data, and the Australian Institute of Health and Welfare (AlHW) as the integrating authority and undertaking the data linkage to the National Death Index (NDI). The authors also acknowledge the following 1) Centre for Health Record Linkage (CHeReL), NSW Ministry of Health and ACT Health for the NSW Admitted Patients and ACT Admitted Patient Care Data Collections; 2) Queensland Health, including the Statistical Services Branch, for the QLD Hospital Admitted Patient Data Collection; 3) Department of Health Western Australia, including the Data Linkage Branch, and the WA Hospital Morbidity Data Collection; 4) SA NT Datalink, SA Health and the Northern Territory Department of Health, for the SA Public Hospital Separations and NT Public Hospital Inpatient Activity Data Collections; 5) The Department of Health Tasmania, and the Tasmanian Data Linkage Unit, for the Public Hospital Admitted Patient Episodes Data Collection; and 6) The Department of Health and Human Services Victoria, Centre for Victorian Data Linkage, for the Victorian Admitted Episodes Dataset. 


\section{References}

1. Rowe H, Holton S, Kirkman M, Bayly C, Jordan L, McNamee K, McBain J, Sinnott V, Fisher J: Prevalence and distribution of unintended pregnancy: the Understanding Fertility Management in Australia National Survey. Aust N Z J Public Health 2016, 40(2):104-109.

2. Davis AR, Pack AM, Kritzer J, Yoon A, Camus A: Reproductive history, sexual behavior and use of contraception in women with epilepsy. Contraception 2008, 77(6):405-409.

3. Chor J, Rankin K, Harwood B, Handler A: Unintended pregnancy and postpartum contraceptive use in women with and without chronic medical disease who experienced a live birth. Contraception 2011, 84(1):57-63.

4. Farahi N, Zolotor A: Recommendations for preconception counseling and care. Am Fam Physician 2013, 88(8):499-506.

5. Chuang $\mathrm{CH}$, Chase GA, Bensyl DM, Weisman CS: Contraceptive use by diabetic and obese women. Womens Health Issues 2005, 15(4):167-173.

6. Perritt JB, Burke A, Jamshidli R, Wang J, Fox M: Contraception counseling, pregnancy intention and contraception use in women with medical problems: an analysis of data from the Maryland Pregnancy Risk Assessment Monitoring System (PRAMS). Contraception 2013, 88(2):263-268.

7. Vahratian A, Barber JS, Lawrence JM, Kim C: Family-planning practices among women with diabetes and overweight and obese women in the 2002 National Survey For Family Growth. Diabetes Care 2009, 32(6):1026-1031.

8. Skinner SR, Smith J, Fenwick J, Hendriks J, Fyfe S, Kendall G: Pregnancy and protection: perceptions, attitudes and experiences of Australian female adolescents. Women Birth 2009, 22(2):50-56.

9. Trussell J, Vaughan B: Contraceptive failure, method-related discontinuation and resumption of use: results from the 1995 National Survey of Family Growth. Fam Plann Perspect 1999, 31(2):64-72, 93.

10. Coombe J, Harris ML, Loxton D: Who uses long-acting reversible contraception? Profile of LARC users in the CUPID cohort. Sex Reprod Healthc 2017, 11:19-24.

11. Rowlands IJ, Mishra GD, Lucke JC: Association between young women's physical and mental health and their method of contraception in a longitudinal, population-based study. BMJ Sex Reprod Health 2021, 47(2):129-136.

12. DeNoble AE, Hall KS, Xu X, Zochowski MK, Piehl K, Dalton VK: Receipt of prescription contraception by commercially insured women with chronic medical conditions. Obstet Gynecol 2014, 123(6):1213-1220.

13. Phillips-Bell GS, Sappenfield W, Robbins CL, Hernandez L: Chronic Diseases and Use of Contraception Among Women at Risk of Unintended Pregnancy. J Womens Health (Larchmt) 2016, 25(12):1262-1269.

14. Harris ML, Egan N, Forder PM, Loxton D: Increased chronic disease prevalence among the younger generation: findings from a population-based data linkage study to inform chronic disease ascertainment among reproductive-aged Australian women. PLoS One 2021, 16(8):e0254668.

15. Harris ML, Coombe J, Forder PM, Lucke JC, Bateson D, Loxton D: Young women's complex patterns of contraceptive use: findings from an Australian cohort study. Perspect Sex Reprod Health 2020, 52(3):181-190.

16. Harris ML, Egan N, Forder PM, Coombe J, Loxton D: Contraceptive use among women through their later reproductive years: findings from an Australian prospective cohort study. PLoS One 2021, 16(8):e0255913.

17. Loxton D, Powers J, Anderson AE, Townsend N, Harris ML, Tuckerman R, Pease S, Mishra G, Byles J: Online and offline recruitment of young women for a longitudinal health survey: findings from the Australian Longitudinal Study on Women's Health 1989-95 cohort. J Med Internet Res 2015, 17(5):e109.

18. Mishra GD, Hockey R, Powers J, Loxton D, Tooth L, Rowlands I, Byles J, Dobson A: Recruitment via the Internet and social networking sites: the 1989-1995 cohort of the Australian Longitudinal Study on Women's Health. J Med Internet Res 2014, 16(12):e279.

19. Pols RG, Hawks DV: Is there a safe level of daily consumption of alcohol for men and women? In. Canberra: National Health and Medical Research Council; 1992.

20. World Health Organization: Obesity: preventing and managing the global epidemic: a report of a WHO consultation. In: WHO technical report series. Geneva, Switzerland: WHO; 2000.

Page $17 / 20$ 
21. Kessler RC: Kessler's 10 Psychological Distress Scale. In. Boston, MA; 1996: Harvard Medical Schoo.

22. Mazza D, Harrison C, Taft A, Brijnath B, Britt H, Hobbs M, Stewart K, Hussainy S: Current contraceptive management in Australian general practice: an analysis of BEACH data. Med J Aust 2012, 197(2):110-114.

23. Australian Government: Department of Health: National Women's Health Strategy: 2020-2030. In. Canberra: Commonwealth of Autralia; 2020.

24. Faculty of Sexual and Reproductive Healthcare Clinical Effectiveness Unit: Faculty of Sexual and Reproductive Healthcare Clinical Guidance Report. In: Contraceptive choices for women with cardiac disease UK: FSRH; 2014.

25. Yazdany J, Trupin L, Kaiser R, Schmajuk G, Gillis JZ, Chakravarty E, Schwarz EB: Contraceptive counseling and use among women with systemic lupus erythematosus: a gap in health care quality? Arthritis Care Res (Hoboken) 2011, 63(3):358-365.

26. Marri SR, Ahn C, Buchman AL: Voluntary childlessness is increased in women with inflammatory bowel disease. Inflamm Bowel Dis 2007, 13(5):591-599.

27. Brito MB, Casqueiro JS, Alves FSS, Lopes JB, Alves R, Santiago M: Low prevalence of contraceptive use among Brazilian women of reproductive age with systemic lupus erythematosus. J Obstet Gynaecol 2018, 38(7):975-978.

28. Dalkilic E, Tufan AN, Oksuz MF, Sahbazlar M, Coskun BN, Seniz N, Pehlivan Y, Inanc M: Comparing female-based contraceptive methods in patients with systemic lupus erythematosus, rheumatoid arthritis and a healthy population. Int $\mathrm{J}$ Rheum Dis 2014, 17(6):653-657.

29. Hibbert EJ, Chalasani S, Kozan P, Myszka R, Park KEJ, Black KI: Preconception care and contraceptive use among Australian women with diabetes mellitus. Aust J Gen Pract 2018, 47(12):877-882.

30. Coombe J, Harris ML, Wigginton B, Lucke J, Loxton D: Contraceptive use at the time of unintended pregnancy: findings from the Contraceptive Use, Pregnancy Intention and Decisions study. Aust Fam Physician 2016, 45(11):842-848.

31. Macintosh MC, Fleming KM, Bailey JA, Doyle P, Modder J, Acolet D, Golightly S, Miller A: Perinatal mortality and congenital anomalies in babies of women with type 1 or type 2 diabetes in England, Wales, and Northern Ireland: population based study. BMJ 2006, 333(7560):177.

32. Peters A, Laffel L, American Diabetes Association Transitions Working G: Diabetes care for emerging adults: recommendations for transition from pediatric to adult diabetes care systems: a position statement of the American Diabetes Association, with representation by the American College of Osteopathic Family Physicians, the American Academy of Pediatrics, the American Association of Clinical Endocrinologists, the American Osteopathic Association, the Centers for Disease Control and Prevention, Children with Diabetes, The Endocrine Society, the International Society for Pediatric and Adolescent Diabetes, Juvenile Diabetes Research Foundation International, the National Diabetes Education Program, and the Pediatric Endocrine Society (formerly Lawson Wilkins Pediatric Endocrine Society). Diabetes Care 2011, 34(11):2477-2485.

33. Birru Talabi M, Clowse MEB, Schwarz EB, Callegari LS, Moreland L, Borrero S: Family planning counseling for women with rheumatic diseases. Arthritis Care Res (Hoboken) 2018, 70(2):169-174.

34. Hinze A, Kutty S, Sayles H, Sandene EK, Meza J, Kugler JD: Reproductive and contraceptive counseling received by adult women with congenital heart disease: a risk-based analysis. Congenit 2013, 8(1):20-31.

35. Gawron LM, Hammond C, Keefer L: Documentation of reproductive health counseling and contraception in women with inflammatory bowel diseases. Patient Educ Couns 2014, 94(1):134-137.

36. Holing EV, Beyer CS, Brown ZA, Connell FA: Why don't women with diabetes plan their pregnancies? Diabetes Care 1998, 21(6):889-895.

37. Chuang $\mathrm{CH}$, Velott DL, Weisman CS: Exploring knowledge and attitudes related to pregnancy and preconception health in women with chronic medical conditions. Maternal \& Child Health Journal 2010, 14(5):713-719.

38. Holton S, Thananjeyan A, Rowe H, Kirkman M, Jordan L, McNamee K, Bayly C, McBain J, Sinnott V, Fisher J: The fertility management experiences of Australian women with a non-communicable chronic disease: findings from the Understanding Fertility Management in Contemporary Australia Survey. Maternal \& Child Health Journal 2018, 22(6):830-840.

39. Eisenberg DL, Stika C, Desai A, Baker D, Yost KJ: Providing contraception for women taking potentially teratogenic medications: a survey of internal medicine physicians' knowledge, attitudes and barriers. J Gen Intern Med 2010, 25(4):291- 
297.

40. Carmine L: Contraception for adolescents with medically complex conditions. Curr Probl Pediatr Adolesc Health Care 2018, 48(12):345-357.

41. Lindley KJ, Conner SN, Cahill AG, Madden T: Contraception and Pregnancy Planning in Women With Congenital Heart Disease. Curr Treat Options Cardiovasc Med 2015, 17(11):50.

42. Yakoob MY, Bateman BT, Ho E, Hernandez-Diaz S, Franklin JM, Goodman JE, Hoban RA: The risk of congenital malformations associated with exposure to beta-blockers early in pregnancy: a meta-analysis. Hypertension 2013, 62(2):375-381.

43. Cooper WO, Hernandez-Diaz S, Arbogast PG, Dudley JA, Dyer S, Gideon PS, Hall K, Ray WA: Major congenital malformations after first-trimester exposure to ACE inhibitors. N Engl J Med 2006, 354(23):2443-2451.

44. Verberne EA, de Haan E, van Tintelen JP, Lindhout D, van Haelst MM: Fetal methotrexate syndrome: A systematic review of case reports. Reprod Toxicol 2019, 87:125-139.

45. Toomey D, Waldron B: Family planning and inflammatory bowel disease: the patient and the practitioner. Fam Pract 2013, 30(1):64-68.

46. Mazza D, Chapman A, Michie S: Barriers to the implementation of preconception care guidelines as perceived by general practitioners: a qualitative study. BMC Health Serv Res 2013, 13:36.

47. eTG complete [digital]. In. Melbourne: Therapeutic Guidelines Limited; 2020 Dec.

48. Coombe J, Harris ML, Loxton D: Examining long-acting reversible contraception non-use among Australian women in their 20s: findings from a qualitative study. Cult Health Sex 2019, 21(7):822-836.

49. Charron-Prochownik D, Ferons-Hannan M, Sereika S, Becker D: Randomized efficacy trial of early preconception counseling for diabetic teens (READY-girls). Diabetes Care 2008, 31(7):1327-1330.

50. Jones RK, Tapales A, Lindberg LD, Frost J: Using Longitudinal Data to Understand Changes in Consistent Contraceptive Use. Perspect Sex Reprod Health 2015, 47(3):131-139.

51. Collins L, Lanza S: Latent class and latent transition analysis. Hoboken, New Jersey: John Wiley \& Sons; 2010.

\section{Figures}




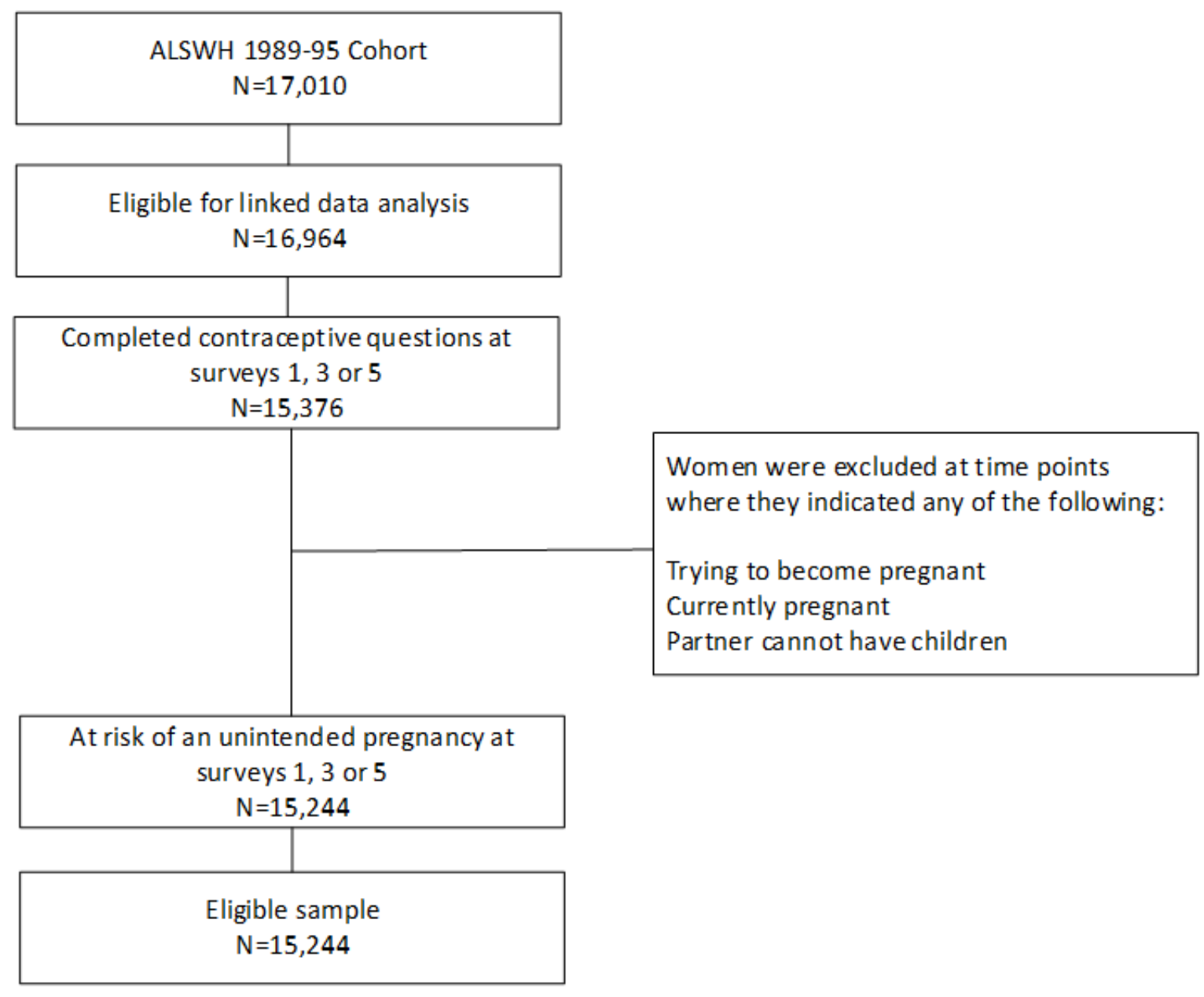

Figure 1

Determination of eligible sample.

\section{Supplementary Files}

This is a list of supplementary files associated with this preprint. Click to download.

- A696NYCSupplementaryMaterial20210818.docx 